\title{
Universiteit
}

Leiden

The Netherlands

\section{Tuning Hydrophobicity of Platinum by Small Changes in Surface Morphology}

Niet, M.J.T.C. van der; Dunnen, A. den; Koper, M.T.M.; Juurlink, L.B.F.

\section{Citation}

Niet, M. J. T. C. van der, Dunnen, A. den, Koper, M. T. M., \& Juurlink, L. B. F. (2011). Tuning Hydrophobicity of Platinum by Small Changes in Surface Morphology. Physical Review Letters, 107(14), 146103. doi:10.1103/PhysRevLett.107.146103

Version: $\quad$ Not Applicable (or Unknown)

License: $\quad$ Leiden University Non-exclusive license

Downloaded from: https://hdl.handle.net/1887/58505

Note: To cite this publication please use the final published version (if applicable). 


\title{
Tuning Hydrophobicity of Platinum by Small Changes in Surface Morphology
}

\author{
Maria J. T. C. van der Niet, Angela den Dunnen, Marc T. M. Koper, and Ludo B. F. Juurlink* \\ Leiden Institute of Chemistry, Leiden University, Einsteinweg 55, P.O. Box 9502, 2300 RA Leiden, The Netherlands
}

(Received 14 March 2011; published 30 September 2011)

\begin{abstract}
Stepped platinum surfaces can become hydrophobic when they are hydrogenated. Even though the $\operatorname{Pt}(533)$ and $\mathrm{Pt}(553)$ surfaces have similar geometries, the hydrophobicity on the deuterated surface is surprisingly different: on $\operatorname{Pt}(533)$ the surface is hydrophobic with water clustering at steps, whereas the entire surface is wet on $\mathrm{Pt}(553)$.
\end{abstract}

DOI: 10.1103/PhysRevLett.107.146103

PACS numbers: 68.08.Bc, 68.43.Hn, 82.45.Jn, 82.65.+r

Groups of water molecules at an interface have the choice between interacting mostly with the substrate or with each other. This competition lies at the heart of a large variety of phenomena with wetting behavior of surfaces likely being the most well-known example. Contact angle studies date at least as far back as Thomas Young's work from the start of the 19th century [1]. Other important areas where such competition governs physical behavior are, e.g., protein folding and micelle formation [2].

For solid surfaces, hydrophilic vs hydrophobic behavior appears difficult to predict. A recent study of water interacting with carbon nanotubes shows that in such confined spaces small changes in temperature may cause a switch between hydrophilic and hydrophobic interaction [3]. Also, minute details of the substrate appear to be of great relevance. A single molecular layer of amorphous solid water is hydrophilic [4], whereas the same layer of crystalline ice is hydrophobic [5].

In this Letter, we show that a small change at the atomic level in substrate morphology without changing chemical identity or confinement size may also affect how water molecules adsorb to a surface. A switch from hydrophobic to hydrophilic behavior is not only apparent from drastic changes in $\mathrm{H}_{2} \mathrm{O}$ 's desorption characteristics, but also in the chemical reactivity toward H-D exchange at well-ordered platinum surfaces. Our results impact on general thinking on long-range ordering of water molecules at interfaces and pose opportunities in tailoring chemistry occurring on nanoparticles as used in, e.g., heterogeneous catalysis and electrochemistry.

As a substrate, we use single crystalline platinum discs, cut and polished to expose either the (533) or (553) surface. Schematic representations of these surfaces for top and side views are shown in Fig. 1 with every circle representing a Pt atom. The only difference between these surfaces is the step type that separates the 4-atom wide (111) terraces. The (533) surface contains the steeper (100) step type, whereas the (553) surface has the more gently sloped (110) step type. The angles that the singleatom high steps make with the terraces are, respectively, $116.6^{\circ}$ and $125.3^{\circ}$. Our platinum surfaces are cleaned and studied under ultrahigh vacuum (UHV) conditions. Details on experimental procedures can be found in the Supplemental Material [6]. Low energy electron diffraction (LEED) confirms the atomic ordering of the surface as depicted in Fig. 1.

The cleaned platinum surfaces are first exposed to $D_{2}$ by background dosing until no more dissociation occurs. To minimize contamination by $\mathrm{H}$, this is done by exposing the single crystals to relatively high $\mathrm{D}_{2}$ pressures while cooling them from $\sim 500 \mathrm{~K}$ to $\sim 150 \mathrm{~K}$. We have previously studied $\mathrm{D}$ adsorption and desorption from these surfaces in detail [7]. Subsequently, $\mathrm{H}_{2} \mathrm{O}$ is dosed onto the D-saturated surface at a temperature $\leq 110 \mathrm{~K}$. We define 1 monolayer (ML) of $\mathrm{H}_{2} \mathrm{O}$ as the sum of the two peaks associated with water desorption from step and terrace sites from the bare (i.e., not D precovered) surfaces in temperature programmed desorption (TPD) experiments [7-9]. Both clean Pt surfaces have been shown to be hydrophilic and both step types stabilize water adsorption relative to the (111) terrace $[7,8,10]$.

Figure 2 shows TPD spectra of water taken at a temperature ramp of $1.0 \mathrm{~K} \mathrm{~s}^{-1}$ corresponding to various amounts of $\mathrm{H}_{2} \mathrm{O}$ dosed onto the D-saturated surfaces. Panels (a) and (b) show the spectra of, respectively, $\mathrm{H}_{2} \mathrm{O}$ and HOD desorbing from $\mathrm{D} / \mathrm{Pt}(533)$, whereas panels (c) and (d) show the same for water desorbing from $\mathrm{D} / \mathrm{Pt}(553)$. In the $\mathrm{H}_{2} \mathrm{O}$ spectra, dashed lines show desorption of water from the bare Pt surfaces. Comparing the $\mathrm{H}_{2} \mathrm{O}$ spectra for the two surfaces, we notice that the desorption traces are remarkably affected by predeuteration, but in dissimilar manners. For both surfaces, the removal of

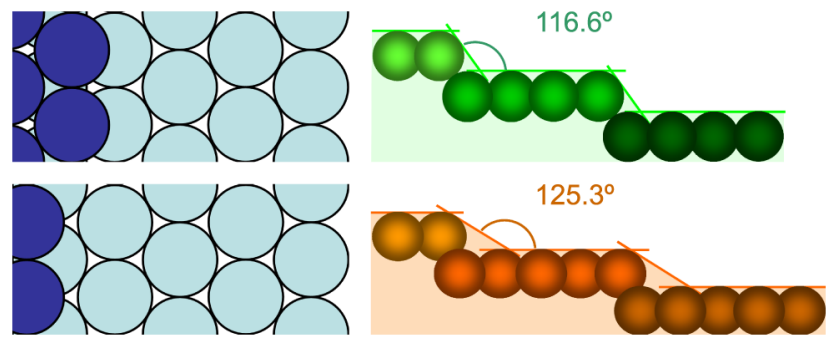

FIG. 1 (color online). Top and side views of (a) $\mathrm{Pt}(533)$ with (100) step type and (b) Pt(553) with (110) step type. 


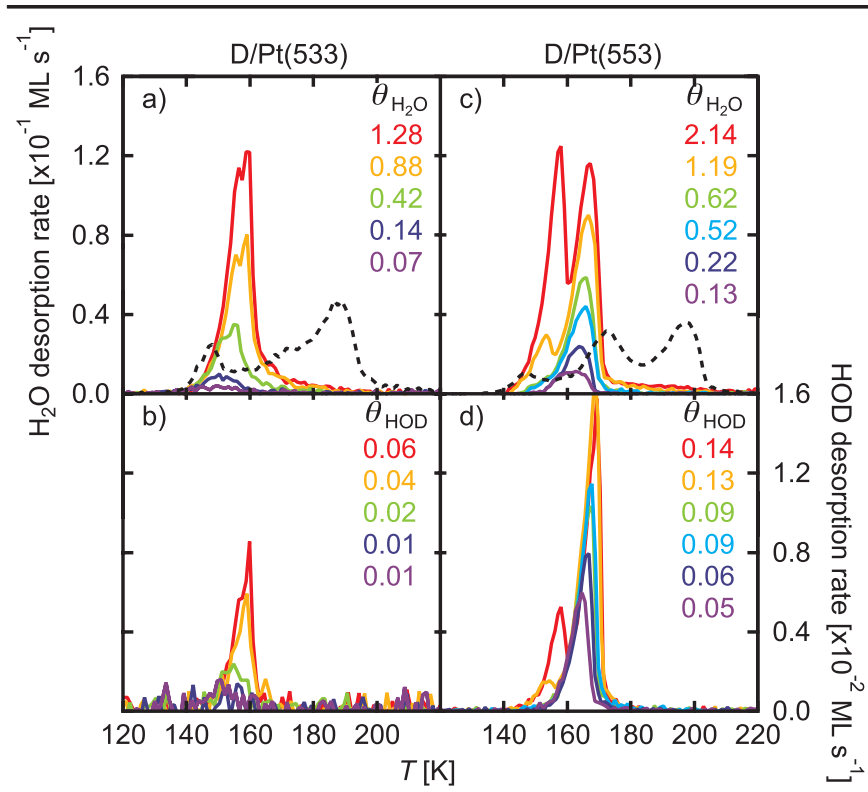

FIG. 2 (color online). TPD spectra of varying amounts of $\mathrm{H}_{2} \mathrm{O}$ and HOD desorbing from $\mathrm{Pt}(533)$ and $\mathrm{Pt}(553)$ surfaces that have been fully precovered with $\mathrm{D}_{\mathrm{ad}}$. (a) $\mathrm{H}_{2} \mathrm{O}$ from $\mathrm{Pt}(533)$, (b) HOD from $\mathrm{Pt}(533)$, (c) $\mathrm{H}_{2} \mathrm{O}$ from $\mathrm{Pt}(553)$, and (d) HOD from $\mathrm{Pt}(553)$. The dashed lines in the $\mathrm{H}_{2} \mathrm{O}$ spectra show $\sim 1$ ML $\mathrm{H}_{2} \mathrm{O}$ desorption from the bare surfaces.

higher temperature peaks at $188 \mathrm{~K}$ for $\mathrm{Pt}(533)$ and $197 \mathrm{~K}$ for $\mathrm{Pt}(553)$ informs us that predeuteration removes the stabilizing effect that steps have on water adsorption (see Ref. [7]). This observation is in contrast to the stabilization of adsorbed water observed by predeuterating $\mathrm{Pt}(111)$ [11]. In fact, on both surfaces the peak temperatures are located below $171 \mathrm{~K}$, indicating a destabilization compared to water adsorption on the (111) terraces [7].

Next, we compare the desorption of water from both deuterated surfaces. Multilayer water desorption is independent of the substrate [12] and starts at $\sim 140 \mathrm{~K} \mathrm{[13].}$ This is exactly what we observe for the single desorption peak visible on $\mathrm{D} / \mathrm{Pt}(533)$ : from the lowest coverages onwards we observe desorption at $140 \mathrm{~K}$. The peak shows typical characteristics of zero order desorption kinetics. Also, the deflection at $\sim 158 \mathrm{~K}$ for $\theta_{\mathrm{H}_{2} \mathrm{O}} \geq 0.42 \mathrm{ML}$ is located at the exact temperature where crystallization from amorphous solid water (ASW) to crystalline ice is observed for $25 \mathrm{ML}$ of ASW on $\mathrm{Pt}(111)[13,14]$. The formation of ASW under these conditions was also concluded from the Reflection Absorption Infrared Spectra we published earlier for this system [9]. The desorption temperature of this peak and the presence of the deflection due to crystallization show that water forms a multilayered structure at submonolayer coverages on $\mathrm{D} / \mathrm{Pt}(533)$. This surface is therefore clearly hydrophobic. Desorption studies on the 6-atom wide D-saturated $\operatorname{Pt}(755)$ surface show identical behavior, indicating that terrace length does not influence hydrophobicity for small terraces separated by (100) steps (see Supplemental Material [6]).
In contrast to the single peak observed on $\mathrm{D} / \mathrm{Pt}(533)$, we clearly observe two peaks for water desorption from $\mathrm{D} / \mathrm{Pt}(553)$ [Fig. 2(c)]. The peak at $151 \mathrm{~K}$ again shows characteristics of zero order desorption kinetics and is located at the same temperature at which water multilayer desorption from bare $\operatorname{Pt}(553)$ starts [7]. Therefore, we ascribe this peak to water multilayer desorption. The other peak at $\sim 162 \mathrm{~K}$ strongly resembles desorption from the bare hydrophilic surface, although it appears at a slightly lowered peak temperature. The size of this feature in comparison to that of $1 \mathrm{ML}_{2} \mathrm{O}$ desorbing from the bare $\mathrm{Pt}(553)$ surface suggests that almost the entire $\mathrm{D} / \mathrm{Pt}(553)$ surface was covered by $\mathrm{H}_{2} \mathrm{O}$. Therefore, $\mathrm{D} / \mathrm{Pt}(553)$ seems to be hydrophilic and the first wetting layer is almost completed prior to formation of the multilayer. We shall return to this point in the discussion of the H-D isotope exchange.

The different interactions between water and these two surfaces are also reflected in the isotope exchange between $\mathrm{H}_{2} \mathrm{O}$ and D. In the HOD signal [Figs. 2(b) and 2(d)], we observe the same structures as for the $\mathrm{H}_{2} \mathrm{O}$ spectra: a single peak for $\mathrm{D} / \mathrm{Pt}(533)$ located at the temperature where water multilayers desorb and a two-peak structure for $\mathrm{D} / \mathrm{Pt}(553)$ indicating two distinguishable water layers of which the first one is nearly completed before the second grows in. Interestingly, we note that on $\mathrm{Pt}(553)$, relatively little HOD desorbs in the multilayer peak compared to the $\mathrm{H}_{2} \mathrm{O}$ spectra. Although the initial exchange between $\mathrm{H}$ and $\mathrm{D}$ atoms to form HOD must obviously take place at the interface, little H-D exchange takes place between the first and subsequent water layers.

The more striking effect however, is the absolute amount of H-D exchange on the two surfaces, especially for low water coverages: $\sim 27 \%$ on $\operatorname{Pt}(553)$ vs only $\sim 8 \%$ on $\mathrm{Pt}(533)$. If the suggested difference in hydrophobic vs hydrophilic character is correct, it provides a straight forward explanation: compared to the more hydrophilic $\mathrm{D} / \mathrm{Pt}(553)$ surface, far less water is in direct contact with the hydrophobic $\mathrm{D} / \mathrm{Pt}(533)$ surface. However, beyond this simple geometric argument, we also note that the spectrum of bond strengths for $\mathrm{D}$ on $\operatorname{Pt}(553)$ reaches lower values than on $\mathrm{Pt}(533)$ [7]. Weaker D-Pt bonds may assist in reaching the $\mathrm{H}_{2} \mathrm{O}+\mathrm{D} \leftrightharpoons \mathrm{HOD}+\mathrm{H}$ equilibrium more rapidly.

The $\mathrm{HD}$ and $\mathrm{D}_{2}$ desorption traces from the same experiments will identify the location of the "droplets" on the hydrophobic $\mathrm{D} / \mathrm{Pt}(533)$ surface and will confirm the formation of a completed "sheet" of water on, and therefore the hydrophilicity of, D/Pt(553). In Fig. 3, we show these desorption traces for $\mathrm{Pt}(533)$ [panel (a)] and $\mathrm{Pt}(553)$ [(panel (b)]. The $\beta_{1}$ and $\beta_{2}$ peaks on $\operatorname{Pt}(533)$ are associated with the recombinative desorption from, respectively, step and terrace sites, whereas on $\operatorname{Pt}(553)$ the $\gamma_{1}$ and $\gamma_{2}$ peaks are associated with desorption from terrace sites and $\gamma_{3}$ is associated with desorption from step sites [7]. As 


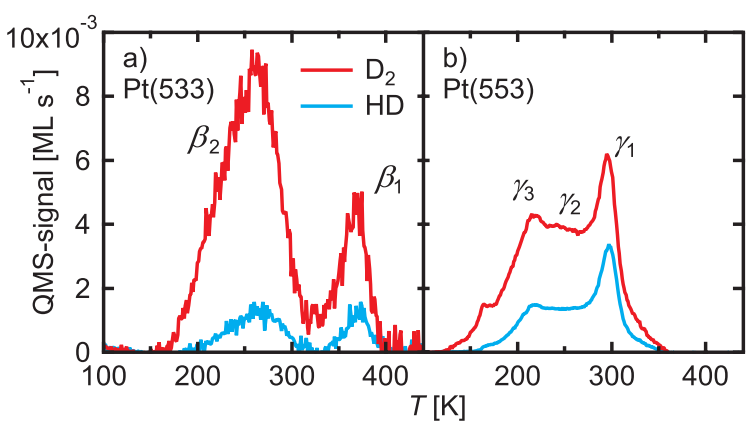

FIG. 3 (color online). TPD spectra of $\mathrm{HD}$ and $\mathrm{D}_{2}$ desorbing from a $\operatorname{Pt}(533)$ surface (a) or $\operatorname{Pt}(553)$ surface (b) that has been fully precovered with $\mathrm{D}_{\text {ad }}$ prior to the adsorption of $\sim 1 \mathrm{ML}$ $\mathrm{H}_{2} \mathrm{O}$.

mentioned, these spectra qualitatively indicate that hydrogen binds weakly to (110) step sites in comparison to (100) steps. A more quantitative comparison of these spectra indicate that on $\mathrm{D} / \mathrm{Pt}(533)$ a clear bias exists for $\mathrm{H}-\mathrm{D}$ exchange in the $\beta_{1}$ peak, i.e., at step sites. We have concluded that on this hydrophobic surface, water "droplets" must therefore be located mostly at steps [9].

For Pt(553), we do not observe such a pronounced bias in Fig. 3. Very little difference in the relative peak heights between the $\mathrm{HD}$ and $\mathrm{D}_{2}$ spectra exist and a simple scaling factor turns one spectrum nearly exactly into the other. Only the $\gamma_{3}$ peak is slightly smaller in the HD spectra compared to the $\mathrm{D}_{2}$ spectra. Therefore, if a bias exists, it is towards exchange on the $\mathrm{D} / \mathrm{Pt}(553)$ terrace sites and $\mathrm{H}_{2} \mathrm{O}$ must have been present at terrace sites at $\theta_{\mathrm{H}_{2} \mathrm{O}} \approx 1 \mathrm{ML}$.

Figure 4 shows the sum of the $\mathrm{HD}$ and $\mathrm{D}_{2}$ TPD spectra from $\mathrm{Pt}(553)$ for various water coverages. We compare

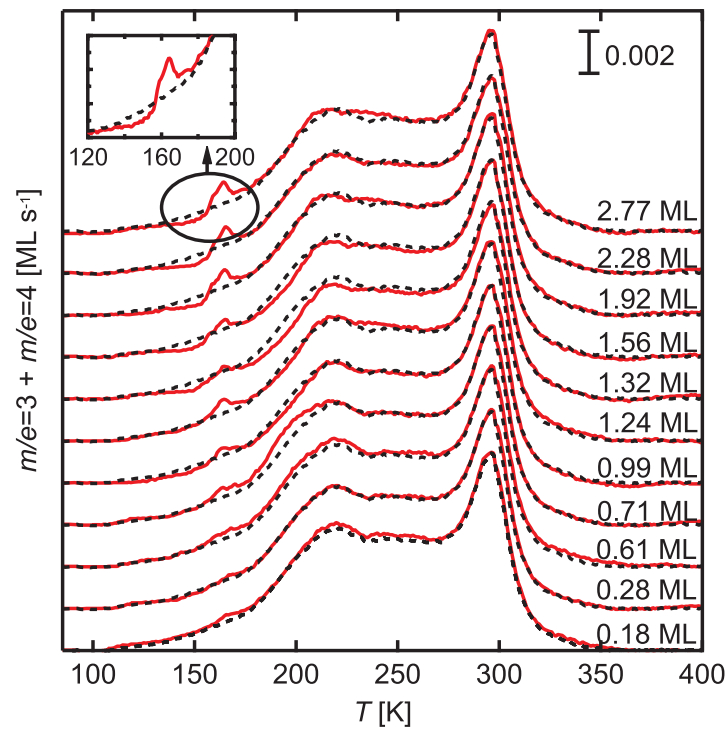

FIG. 4 (color online). TPD spectra of $\mathrm{HD}$ and $\mathrm{D}_{2}$ desorbing from a $\operatorname{Pt}(553)$ surface that has been fully precovered with $\mathrm{D}_{\mathrm{ad}}$ after which varying amounts of $\mathrm{H}_{2} \mathrm{O}$ were dosed on the surface. The dashed lines show $1 \mathrm{ML} \mathrm{D}_{\mathrm{ad}}$ desorbing from bare $\operatorname{Pt}(553)$. every trace to $\mathrm{D}_{2}$ desorbing from bare $\mathrm{Pt}(553)$ (dashed lines). The coadsorption TPD spectra are very similar to those on bare $\mathrm{Pt}(553)$. The inset in Fig. 4 shows that for the highest $\theta_{\mathrm{H}_{2} \mathrm{O}}$, the only difference is a minor but significant delay in deuterium desorption from the $\mathrm{H}_{2} \mathrm{O}$ post-covered surface followed by a small additional peak at $163 \mathrm{~K}$. At higher temperatures, the spectra are identical within our margin of error. The onset of deuterium desorption in the inset coincides with the start of water desorption in the high temperature peak in the corresponding water TPD spectra of Fig. 2. This behavior is reminiscent of the "molecular volcano" observed for ASW adsorbed on top of $\mathrm{CCl}_{4}$ on $\mathrm{Au}(111)$, where $\mathrm{CCl}_{4}$ desorption is blocked by the ASW overlayer and occurs abruptly when cracks are formed in the overlayer when the ASW crystalizes [15]. We explain our observation as follows: once the water from our overlayer starts to desorb, the underlying $D_{a d}$ can associatively desorb, causing a temporarily higher $\mathrm{D}_{2}$ desorption rate compared to the bare surface. The formation or desorption of $\mathrm{D}_{2}$ is inhibited by (less than) a single monolayer of water.

For $\theta_{\mathrm{H}_{2} \mathrm{O}} \leq 1.92 \mathrm{ML}$ the additional peak at $163 \mathrm{~K}$ becomes less pronounced and shifts to higher temperatures with decreasing water coverage, following the zero order desorption kinetics of the corresponding $\mathrm{H}_{2} \mathrm{O}$ spectra. However, the additional peak remains visible for the lowest $\mathrm{H}_{2} \mathrm{O}$ coverages. This implies that at low coverages $\mathrm{H}_{2} \mathrm{O}$ is preferentially adsorbed at the sites associated with $\mathrm{D}_{2}$ desorption in $\gamma_{3}$, which are (110) step sites. Since we also concluded that at $1 \mathrm{ML}_{2} \mathrm{O}$ is present at terraces and the peak at $162 \mathrm{~K}$ is of a size of $\sim 1 \mathrm{ML}, \mathrm{H}_{2} \mathrm{O}$ must be present across the entire $\mathrm{Pt}(553)$ surface. The $\mathrm{H}_{2} \mathrm{O}$ overlayer appears to grow from the steps onto the terraces, ultimately forming a complete single layer "sheet" of water. On the $\operatorname{Pt}(533)$ surface, water initially wets (part of) the step sites, but then grows three-dimensional clusters without wetting the terraces fully [9]. As mentioned, the formation of these three-dimensional clusters is in agreement with our earlier RAIRS measurements [9]. We also note that we have performed LEED studies on $\mathrm{H}_{2} \mathrm{O}$ and $\mathrm{H}_{2} \mathrm{O}+\mathrm{D}$ coadsorbed systems. LEED images unfortunately yield no information as they show no more than the split-spot patterns characteristic of the Pt substrates published previously [9].

In providing clues for a plausible interpretation of this unanticipated difference in hydrophilicity of deuterated $\operatorname{Pt}(533)$ and $\operatorname{Pt}(553)$, let us outline the main geometrical and energetic differences between these two surfaces, in the absence and presence of deuteration, realizing that ultimately also geometrical differences must impact on the energetics of water adsorption to explain the differences in hydrophilicity.

As the main difference between the two surfaces is the step geometry, it seems likely that the structure of water at step edges is different. For water adsorbing on undeuterated 
Pt, STM studies have shown that on (100) step edges, water initially forms one-dimensional chains along the steps, whereas for (110) steps these are not clearly adsorbed [16]. On deuterium-covered Pt, the interaction of water with such surfaces will be driven by the (in)ability of water to form relatively stable two-dimensional hydrogen-bonded networks extending onto the terraces, versus the tendency of water to be structured into solvation shells of the adsorbed deuterium. We note that from density-functional theory (DFT) calculations, the interaction of water layers with $\mathrm{Pt}(111)$, i.e., the water-Pt bonding, is known to be very weak and its stability on such surfaces is mainly determined by the two-dimensional hydrogen bonding network [16]. Therefore, we expect that the fact that adsorbed deuterium inhibits the direct interaction of water with the platinum surface atoms, will have only a minor effect on its stability. Apparently, hydrogen in a (110) step edge on platinum causes a corrugative smoothening in terms of water adsorption, whereas hydrogen in a (100) step edge leads to an enhanced corrugation causing localized growth of water "droplets" near or at the step sites. Perhaps this could be related to the steepness of the step site (see Fig. 1), but we would like to point out another difference in the properties on these two surfaces related to their interaction with atomic hydrogen in the absence of water. The preferred binding site of hydrogen and deuterium at the (100) step site appears to be the bridge site between two Pt atoms along the step (i.e., the two dark blue step atoms in the top panel of Fig. 1), according to DFT calculations of the similar Pt(211) surface [17]. The same step-bridge site has been computed to be the preferred binding site on $\operatorname{Pt}(331)$, a stepped surface with a (110)-type step, but with the interesting difference that the binding energy difference between step-bound hydrogen and terrace-bound hydrogen is larger for the (100)-type step than for the (110)-type step [18]. In fact, according to the DFT calculations, the $\mathrm{H}$ binding energy surface on $\mathrm{Pt}(331)$ with a (110)-type step is almost flat, whereas $\mathrm{H}$ clearly prefers the step site on $\mathrm{Pt}(211)$ with a (100) step [18]. This agrees qualitatively well with our UHV experiments that show deuterium binds stronger to (100) step sites in $\mathrm{Pt}(533)$ than to (110) step sites in $\mathrm{Pt}(553)$, even to the extent that we concluded from TPD experiments that on Pt(553) terraces are preferred binding sites for deuterium compared to step sites [7]. If the more strongly bound hydrogen somehow acts as an anchoring point for water molecules, than this could relate to the induced corrugation of $\mathrm{Pt}(533)$ in the presence of adsorbed hydrogen.

Although at this moment we can really only speculate about the exact molecular origin of the observed differences in wetting of deuterium-covered $\operatorname{Pt}(553)$ and $\operatorname{Pt}(533)$, our results impact on the molecular thinking of interfacial water. The experiments discussed herein show that small changes in the atomic-level structure of edges and steps may have a dramatic effect on how water organizes around such surface perturbations, leading to either two-dimensional wetting or three-dimensional cluster growth. This may influence the chemical reactivity of such interfaces, as clearly demonstrated by the delay in $\mathrm{D}_{2}$ associative desorption from $\operatorname{Pt}(553)$ when the surface is (partially) covered by a wetting layer of water, whereas no such phenomenon is observed on $\mathrm{Pt}(533)$. In a related though admittedly more remote setting, these effects may affect the reactivity of platinum under aqueous electrochemical conditions, since step sites often feature as the active sites for many catalytic reactions, as for instance taking place in fuel cells [19].

This work was supported financially by the Netherlands Organization for Scientific Research (NWO).

*1.juurlink@chem.leidenuniv.nl

[1] T. Young, Phil. Trans. R. Soc. London 95, 65 (1805).

[2] D. Chandler, Nature (London) 437, 640 (2005).

[3] H.-J. Wang, X.-K. Xi, A. Kleinhammes, and Y. Wu, Science 322, 80 (2008).

[4] G. A. Kimmel, N. G. Petrik, Z. Dohnálek, and B. D. Kay, J. Chem. Phys. 125, 044713 (2006).

[5] G. A. Kimmel, N. G. Petrik, Z. Dohnálek, and B. D. Kay, Phys. Rev. Lett. 95, 166102 (2005).

[6] See Supplemental Material at http://link.aps.org/ supplemental/10.1103/PhysRevLett.107.146103 for experimental details and results for desorption from $\mathrm{Pt}(755)$.

[7] M. J. T. C. van der Niet, A. den Dunnen, L. B. F. Juurlink, and M. T. M. Koper, J. Chem. Phys. 132, 174705 (2010).

[8] M. L. Grecea, E. H. G. Backus, B. Riedmüller, A. Eichler, A. W. Kleyn, and M. Bonn, J. Phys. Chem. B 108, 12575 (2004).

[9] M. J. T. C. van der Niet, I. Dominicus, M. T. M. Koper, and L. B. F. Juurlink, Phys. Chem. Chem. Phys. 10, 7169 (2008).

[10] D. C. Skelton, R. G. Tobin, G. B. Fisher, D. K. Lambert, and C. L. DiMaggio, J. Phys. Chem. B104, 548 (2000).

[11] N. G. Petrik and G. A. Kimmel, J. Chem. Phys. 121, 3727 (2004).

[12] R. S. Smith, C. Huang, E. K. L. Wong, and B. D. Kay, Surf. Sci. 367, L13 (1996).

[13] P. Löfgren, P. Ahlströhm, D. V. Chakarov, J. Lausmaa, and B. Kasemo, Surf. Sci. 367, L19 (1996).

[14] J. L. Daschbach, B. M. Peden, R. S. Smith, and B. D. Kay, J. Chem. Phys. 120, 1516 (2004).

[15] R.S. Smith, C. Huang, E. K. L. Wong, and B. D. Kay, Phys. Rev. Lett. 79, 909 (1997).

[16] P. Vassilev, R. A. van Santen, and M. T. M. Koper, J. Chem. Phys. 122, 054701 (2005).

[17] R. A. Olsen, S. C. Badescu, S. C. Ying, and E. J. Baerends, J. Chem. Phys. 120, 11852 (2004).

[18] T. Vehviläinen, P. Salo, T. Ala-Nissila, and S.C. Ying, Phys. Rev. B 80, 035403 (2009).

[19] M. T. M. Koper, Nanoscale 3, 2054 (2011). 